\title{
Design and Fabrication of Tri-Stopband Bandstop Filters Using Cascaded and Multi-Armed Methods
}

\author{
Ahmed Boutejdar ${ }^{1}$, Saad Dosse Bennani ${ }^{2}$ \\ ${ }^{1}$ German Research Foundation DFG, Electrical Engineering, Bonn-Braunschweig, Germany \\ ${ }^{2}$ Communication Engineering, University Sidi Mohamed Ben Abdellah, Fès, Morocco \\ *corresponding author, E-mail: boutejdar69@gmail.com
}

\begin{abstract}
In this paper, we proposed a compact C-open-loop ring resonator and its equivalent circuit. The second cascaded bandstop filter (BSF) are designed using this simple C-ring resonator. The double ring BSF consists of two cascaded Cring resonators, which are placed on the $\mathrm{RO} 4003$ substrate, while the other triple BSF structure consists of tree cascaded C-ring resonators, which are connected with input and output through microstrip feed lines. The both filters are simulated, optimized and partially realized using MWR simulator and Anritsu E5072A vector network analyzer VNA. In order to reduce the size and to improve the filter characteristics, novel compact filter topologies are designed basing on the previous structures. The proposed multi-band BSFs consist of several open-loop ring resonators placed vertically overlapping (coupled multi-armed ring resonator). Using this idea, the filter topologies with design flexibility, close size and excellent results are reached. The novel compact multi-band BSFs produce several stopband along a frequency range from $\mathrm{DC}$ to $9 \mathrm{GHz}$, in which each separate band exhibits an acceptable and useful bandwidth. Each stopband has regenerated two reflexion zeros, what leads to a good sharpness factors in the transition domains. Good agreement between the experimental results, full-wave simulation has been achieved. Such compact and flexible filters can be successfully used in several microwave and in modern wireless and mobile/satellite communication systems.
\end{abstract}

\section{Introduction}

In recent years, the demand for compact microstrip filters is increasing due to the recently expanding microwave and mobile communication systems. So far, a few effective techniques have been successfully developed to miniaturize the filter size and to transform a simple filter structure to multi-band filter without additional components. Nowadays multi-standard wireless communication systems require multi-band operating transceivers. Dual-band and multi-band filters are in demand for these wireless systems. Beside the multi-band operation, low cost, simplicity, small occupied area and performance are desired features for these filters. Over these years, microstrip planar multi-band filters have been widely proposed as an approach solution to solving the most difficult technical challenges in microwave and wireless communication areas [1]-[3]. In order to increase the number of bands of a structure, they were firstly implemented by the cascaded two filters at different frequencies, with the inherent disadvantage of increased total size [3]. Also they were realized by using a combination of a wide-band bandpass filter and a bandstop filter [4]. However, the size of topology remained a big challenger. Alternatively, other techniques as filter with open stubs are used to regenerate transmission zeros in order to separate the passbands and lead to create several separate bands [3], [5]. Stepped-impedance resonators (SIR) are utilized to realize the dual-stopband characteristics, and reduce size of filters. Stepped impedance resonator (SIR) can adjust the second passband by regulation the impedance ratio and electric lengths of SIRs. By correctly choosing the relevant impedance or strip width ratio, the dual-band topologies employing SIRs can be produced [6]. On the other hand, few researchers have investigated the design of tripleband filters. Boutejdar, et. al., Ren, et. al. and Hejazi, et. al. proposed filter using vertical and horizontal cascaded DGSResonators [7]-[11] and hairpin slots [12], [13]. The disadvantages in this works are the complicated used DGS shapes, the undesired coupling between the DGS resonators and the large size of the used filter structures. Quendo, et. al. proposed the dual- behavior resonators as good candidate for the design of tri-band filters [14]. Mokhtari, et. al. and Boutejdar et. al. proposed a design methodology to these kind of filters using cross-coupled networks represented by coupling matrix [15]-[18]. However, in both proposed topologies, the disadvantage of large circuit size remains a real challenge. In [15], the coupling of two tri-band dualbehavior resonators DBRs was needed to achieve a second order triple-band filter response with six poles (two poles in each band) and five transmission zeros (single zero between each transmission band and two transmission zeros to the left and to the right of all passbands). This cascaded topology shows an undesired increase in the size of the proposed filter. In [15], six hairpin resonators were needed to achieve a filter with triple-band features with six poles (two in each band) and four transmission zeros (two transmission zeros between each two bands).

In this paper, we present BSF structures using open loop ring resonators and overlapping technique. The First one is a simple BSF employing single C-open-loop resonator. Basing 
on this Ring resonator, a double and a triple ring BSFs are designed, simulated and optimized. In order to eliminate the advantages of big size and to improve the characteristics of this topologies, a new compact multi-band microstrip BSFs are proposed using a novel multi-reject band resonator. The idea was, the design of filter topology, which consists of several open-loop ring resonators placed vertically overlapping, and thus to reach filter with design flexibility, close size and satisfactory results. The compact filters are designed, fabricated and measured. The comparison between results shows a reasonable agreement between the measured and simulated responses.

\section{Basic cell of the compact C-open-loop BSF}

The building block of the proposed BSF is a C-open-loop BSF employing a single microstrip stepped open loop resonator that is directly connected with the two feed Lines. Its layout is shown in Fig. 1. The high impedance line between the two feed lines has a constant width corresponding to an impedance of $75 \Omega$. The BSF has been designed at a center frequency of around $5.6 \mathrm{GHz}$, and was simulated and fabricated on a substrate with a relative dielectric constant $\varepsilon_{\mathrm{r}}=3.38$ and a thickness of $0.813 \mathrm{~mm}$. The dimensions of the proposed filter are as follows: $1_{1}=8$ $\mathrm{mm}, \mathrm{l}_{2}=7.6 \mathrm{~mm}, \mathrm{l}_{3}=4.08 \mathrm{~mm}, \mathrm{w}=1.92 \mathrm{~mm}, \mathrm{w}_{1}=1 \mathrm{~mm}, \mathrm{~g}$ $=1 \mathrm{~mm}, \mathrm{t}=2 \mathrm{~mm}$. The two feed lines have a characteristic impedance of $50 \Omega$. The Fig. 2 shows the fabricated structure cell (single BSF).

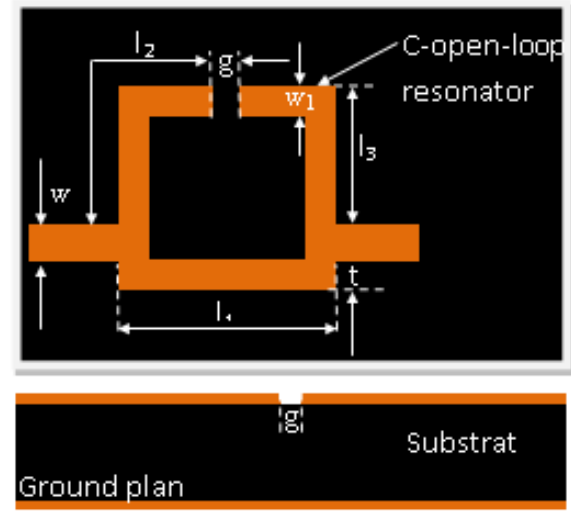

Figure 1. Layout of the C-open-loop single BSF.

The results of EM simulation, equivalent circuit and measurements are all shown in Fig. 3. The equivalent circuit of this $\mathrm{C}$ open loop resonator consists of a capacitor corresponds to the gap of the $\mathrm{C}$ open ring and an inductance, which presents the microstrip arms of $\mathrm{C}$ ring (Fig. 4). Using empirical method, the required dimensions of the ring resonator are chosen. Basing on the scattering responses and the below equations, the values of this intuitive equivalent circuit are calculated. The equivalent circuit presents in Fig. 3 is reduced to the new compact equivalent circuit, as the Fig. 4 shows. The characteristics frequencies can easy extracted from the S-parameters of the ring resonator. The good agreement among them demonstrates the validity of equivalent circuit model for the proposed BSF. As can be seen from the results, the insertion loss from $\mathrm{DC}$ to $3 \mathrm{GHz}$ is less than $0.5 \mathrm{~dB}$, the return loss is less than $-22 \mathrm{~dB}$ in the pass band. The stopband rejection levels are larger than 20 dB from 4.7 GHz to $6.7 \mathrm{GHz}$. The proposed filter exhibits sharp cutoff dropping from less than $-0.5 \mathrm{~dB}$ to almost -44 $\mathrm{dB}$ within a range of $1.5 \mathrm{GHz}$, The size of the proposed $\mathrm{BSF}$ is $7 \times 7 \mathrm{~mm}^{2}$, as Fig. 2. shows.

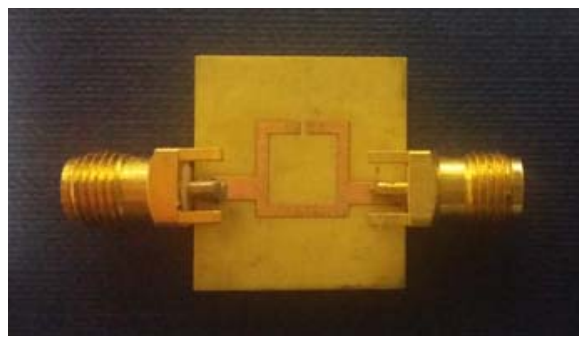

Figure 2. Photograph of fabricated C-open-loop resonator (BSF).

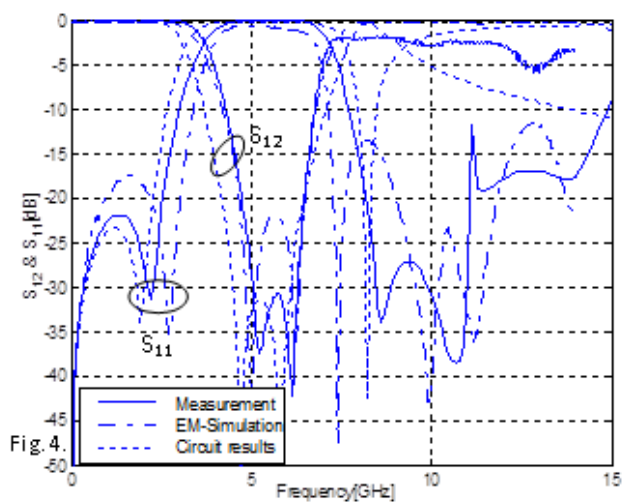

Figure 3. Measured and simulated scattering S-parameter results of C-open-loop resonator (BSF).
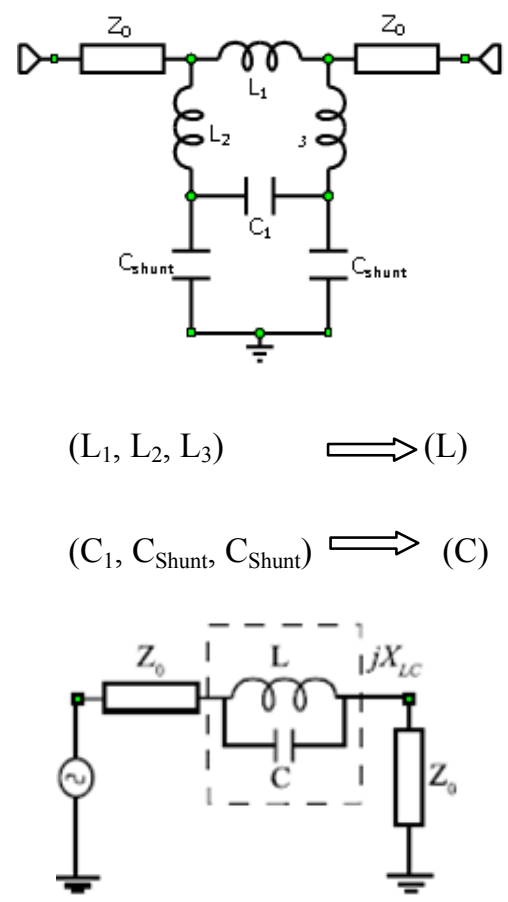

Fig. 4. Equivalent circuit of the C-open-loop resonator . 
$\mathrm{Z}_{0}$ is the input and output impedance, $\mathrm{L}$ is the equivalent inductance and $\mathrm{C}$ is the equivalent capacitance of the resonator-element. The reactance $\mathrm{X}_{\mathrm{LC}}$, which corresponds to the equivalent circuit, is calculated as follows:

$$
\begin{aligned}
& j X_{L C}=\frac{j \omega L\left(\frac{1}{j \omega C}\right)}{j \omega L+\frac{1}{j \omega C}}=\frac{j \omega L}{1-\omega^{2} L C} \\
& X_{L C}=\frac{1}{\omega_{0} C\left(\frac{\omega_{0}}{\omega}-\frac{\omega}{\omega_{0}}\right)} \text { mit }=\omega^{2}=\frac{1}{L C}
\end{aligned}
$$

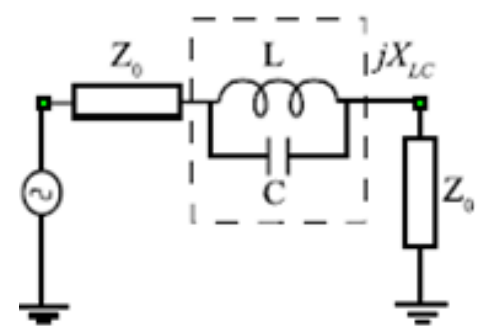

Figure 5. First order Butterworth BSF.

$$
L=\frac{g_{1} Z_{0}}{\omega_{c}} \Rightarrow \omega L=X_{L}=g_{1} Z_{0} \frac{\omega}{\omega_{c}}=g_{1} Z_{0} \omega^{\prime}
$$

With $Z_{0}$ as scaled characteristic impedance of the input and output port and $\mathrm{g}_{1}=2$ as a prototype value of the Butterworth low-pass filter first order. In order to extract the values for the equivalent circuit elements of the DGSresonator [19]-[26]. It is to equal at certain frequency the characteristics of the equivalent circuit of the DGS with that of the prototype low pass filter (Fig. 5). In this case, the frequency will be selected as cutoff frequency $f_{c}$.

$$
\left.X_{L C}\right|_{\omega=\omega_{c}}=\left.X_{L}\right|_{\omega^{\prime}=1}
$$

Thus, the parallel capacitance of the equivalent circuit can calculate as following:

$$
C=\left.\frac{1}{X_{L C}\left(\frac{\omega_{0}}{\omega}-\frac{\omega}{\omega_{0}}\right) \omega_{0}}\right|_{\omega=\omega_{c}}=\frac{\omega_{c}}{g_{1} Z_{0}\left(\omega_{0}^{2}-\omega_{c}^{2}\right)}
$$

The lumped elements of the parallel resonator are defined as following:

$$
C=\frac{5 f_{c}}{\pi Z_{0}\left(f_{0}^{2}-f_{c}^{2}\right)} p F
$$

$$
L=\left[\frac{250}{C\left(\pi f_{0}\right)^{2}}\right] n H
$$

For the frequency range $\mathrm{f}<\mathrm{f}_{0}$, the DGS-element behaves inductively. The value of the equivalent inductance $\mathrm{L}_{\mathrm{eq}}$ for this frequency range is calculated as follows,

$$
L_{e q}=\frac{L}{\left[1-\left(\frac{f}{f_{0}}\right)^{2}\right]} n H
$$

\section{Design of the improved cascaded two rings BSF}

In order to widen the reject band of this filter, without changing the center frequency, we propose a cascaded filter which is composed of two identical C-open-loop cells, as shown in Fig. 6. The suggested filter consists of two Copen-loop resonators, which are placed on the top layer and connected together with a $50 \Omega$ microstrip line. This double cell BSF has better performance, since it suppresses ripples and has a stop band twice as large as the single loop filter. As the Fig. 7 demonstrated, the investigated double-C BSF has a rejection level better than $20 \mathrm{~dB}$ from 4 to $7.2 \mathrm{GHz}$. The use of the introduced series resonant elements allows a sharp cutoff frequency response and a stopband, which is nearly two times broader than the reject band of simple Celement. The second harmonic is in this case suppressed. The both results are presented with good agreement as can be seen from Figs. 3, 4. The disadvantage of this structure is the limited application. The idea here is find an optimization method for generating multi-band characteristics without using complicated topology.

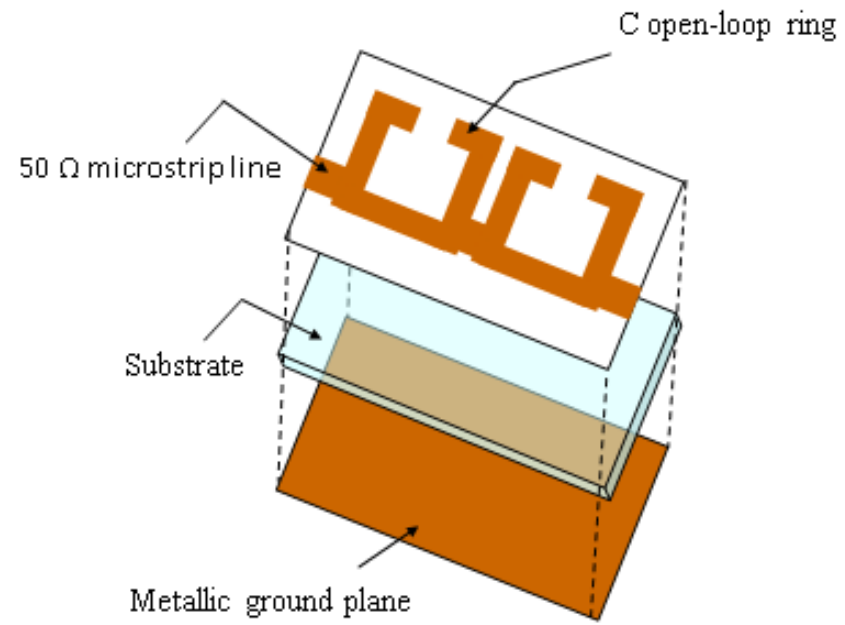

Figure 6. 3D-view of cascaded two rings BSF. 


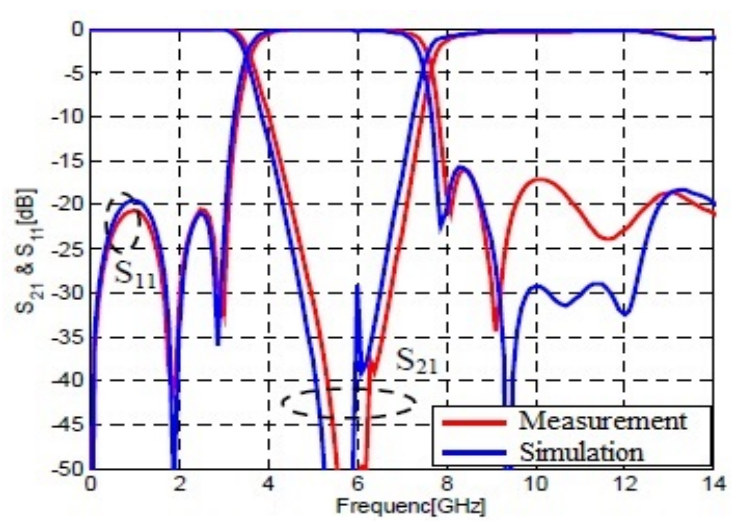

Fig. 7. Measured and simulated scattering S-parameter results of cascaded two rings BSF.

\section{Distribution of magnetic field at pass-band and stop-band frequency ranges}

The aim of this experiment is to demonstrate, basing on behaviors of surface electric and magnetic energies, the relationship between the EM-simulation results and the field distribution of the proposed open-loop ring BSF. The energy distribution is simulated at two different frequencies in the pass- and stop-band. Fig. 8(a) shows the field distribution in the stop-band region at the frequency of 5.5 $\mathrm{GHz}$. At this frequency, the flux-energy is blocked at the input of the structure. At the same time the magnetic energy is distributed along of the ring-arm while the electric energy is focused near of the gab of ring resonator, which indicated that the structure is in the stopband state, more precisely, the structure undergoes a resonance effect as Fig. 8(a) shows. Around $2 \mathrm{GHz}$ almost the whole RF magnetic energy was transmitted from the input to the output using the under metal arms of the cascaded ring resonators. The bridge-arms are direct connected with the $50 \Omega$-feed lines. A negligible electric energy is appeared between in the gap (no metal), what signified that the filter is in the passband state and the metal strip has inductance behavior Fig. 8(b). Finally, it can be easily extracted that the ring structure corresponds then to a parallel LC-resonator.
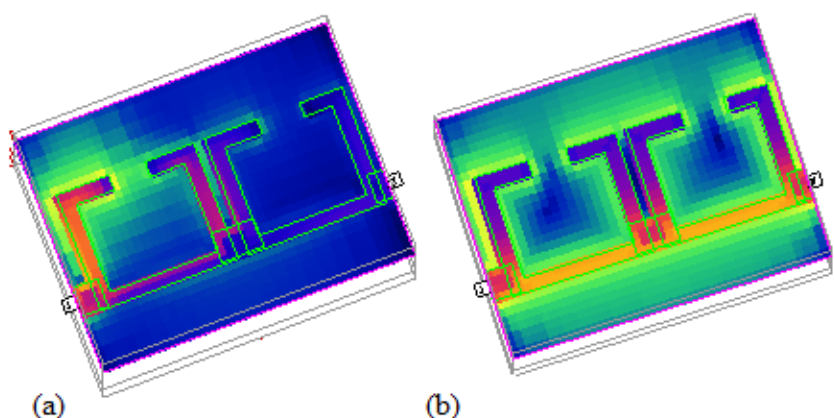

(b)

Figure 8. EM field distribution. (a) $\mathrm{f}=5.5 \mathrm{GHz}$, (b) $\mathrm{f}=2$ $\mathrm{GHz}$.

\section{Design of the improved cascaded three rings BSF}

This new cascaded BSF is proposed with the aim to sharpen the transition from the stopband to passband and try to make appear the harmonic signals thus several stop bands. As shown in Fig. 9, the filter consist of three cascaded mixed coupled C-ring-resonators, in which the first and the third are connected with the $50 \Omega$ feed lines. The three resonators are located on the top layer and direct connected each other. The three microstrip resonators are identical. The filter was simulated for a Rogers RO4003 substrate with a relative dielectric constant of $r_{\varepsilon}=3.38$ and a thickness of $h=0.813$ $\mathrm{mm}$. The BSF was designed to have center frequency at $\mathrm{f}_{0}=$ $3.5 \mathrm{GHz}$ and a cutoff frequency of $\mathrm{f}_{\mathrm{c}}=2.4 \mathrm{GHz}$. The simulation results of the BSF are shown in Fig. 10. The simulated insertion loss and return loss as well as the other stopbands are not satisfactory as shown the Fig. 10. Thus, in order to reduce the filter size and to improve the multiband characteristics of this BSF, another new topology with other layout procedure will be used.

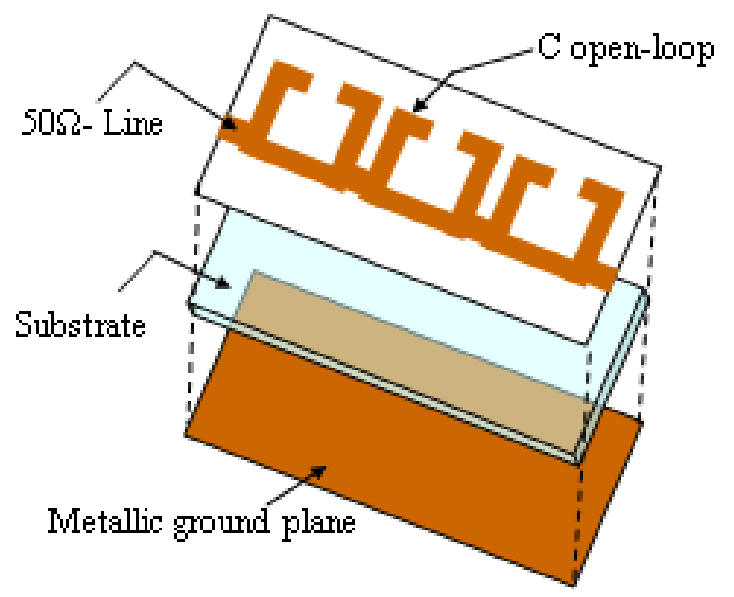

Figure 9. 3D-view of cascaded triple rings BSF.

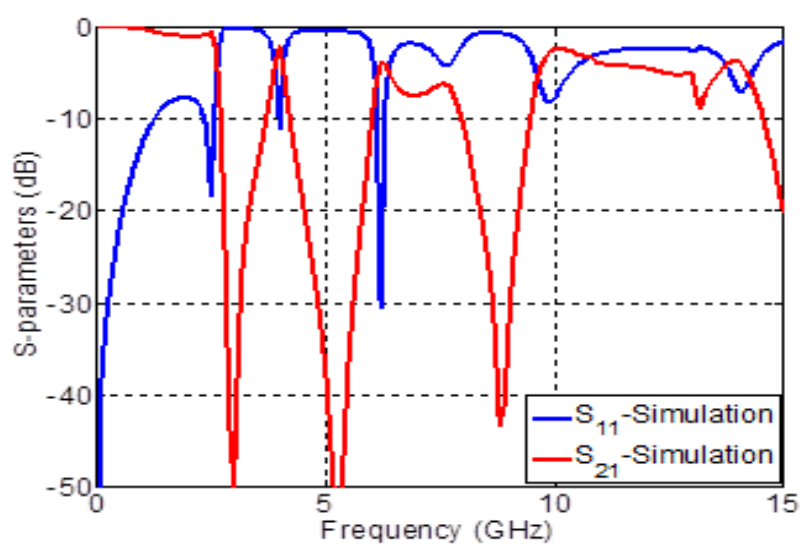

Figure 10. Measured and simulated scattering S-parameter results of cascaded triple rings BSF. 


\section{Design of Dual-band BSF using double-armed open loop resonator}

In order to improve the features of the proposed BSF and thus the creation of dual band along the frequency domain, a new topology is proposed, in which the $\mathrm{C}$ ring resonators are vertically placed on the top of layer of the substrate. As the Figs. 11 and 12 show, the used resonators are etched on the top layer and placed behind each other in vertical direction (vertically overlapping).

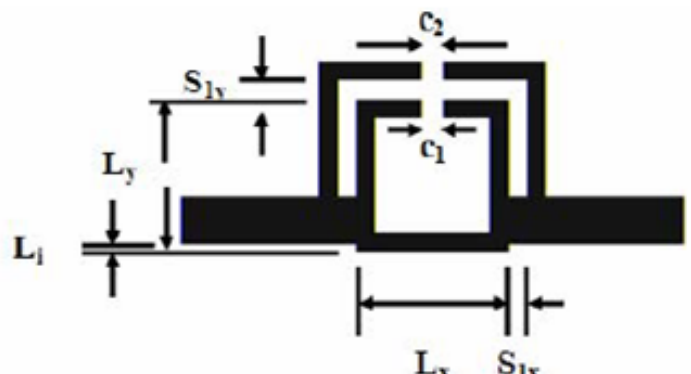

Figure 11. Layout of the improved double rings BSF (Sample I).

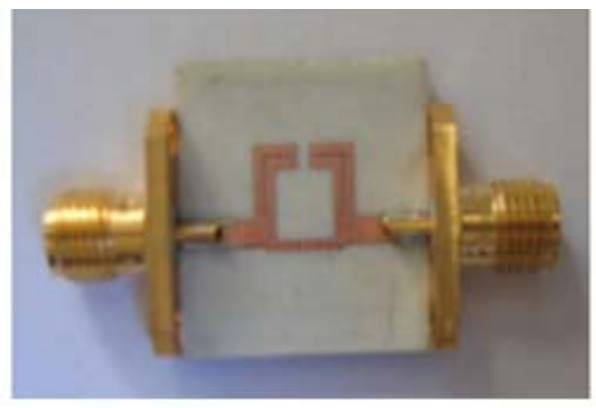

Figure 12. Photograph of the fabricated BSF (Sample I).

The idea of multi-armed open loop resonator is believed to be valid for more than double pair of arms, with the aim to regenerate several stopbands (Fig. 13). Hence, triple armed structure, shown in Fig. 14, was investigated to validate its generalization. Unfortunately, increasing of resonator number leads to design complication and uncontrolled coupling of the topology arms and thus the features of periodicity can be broken. The proposed double ring BSF is designed, simulated, optimized and fabricated on RO4003c substrate. The fabricated filter is shown in Fig. 12. The dimensions of this structure are chosen, basing on the simple $\mathrm{C}$-open-loop ring resonator and the empirical method. All dimensions are tabulated in Table I. Fig. 13 shows the filter responses that were measured on an Anritsu E5072A vector network analyzer VNA, versus simulated response. The fabricated filter exhibits a very good response in terms of the reject-band. The filter also has a reflexion zeros near to the stopband edges at $3.7 \mathrm{GHz}$ and $7.2 \mathrm{GHz}$, which lead to a high sharpness factor in transition domains. Also, the return loss within the stopbands is less than $1 \mathrm{~dB}$. The shift in frequency between the measured and simulated responses is believed to be due to the fabrication tolerance at our facilities.

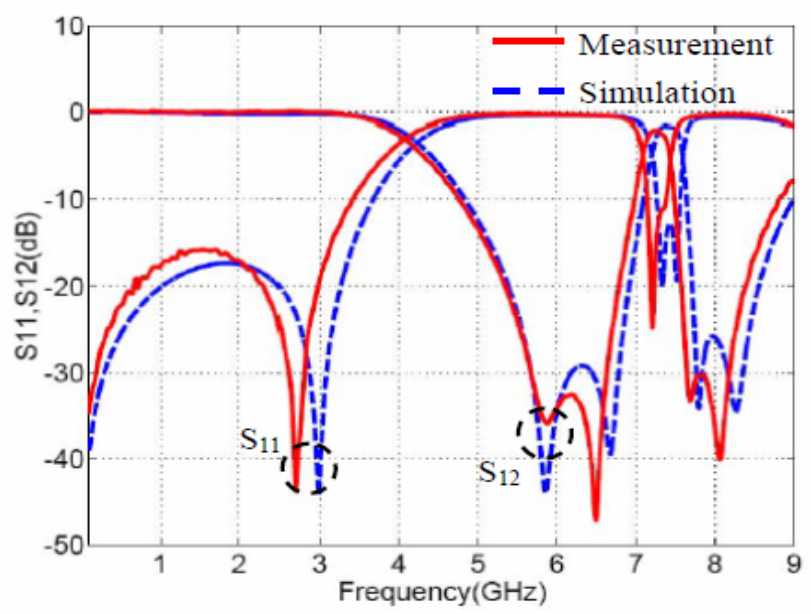

Figure 13. Measured and simulated scattering S-parameter results of double rings BSF (Sample I).

Table 1. Dimensions of proposed BSF.

\begin{tabular}{|c|c|c|}
\hline Dim $\mathrm{mm}$ & Sample I & Sample II \\
\hline$L_{x}$ & 6.00 & 6.00 \\
\hline$L_{y}$ & 6.00 & 6.00 \\
\hline$L_{i}$ & 0.30 & 0.30 \\
\hline$c_{1}$ & 0.90 & 0.90 \\
\hline$S_{1 x}$ & 0.20 & 0.20 \\
\hline$S_{1 y}$ & 0.20 & 0.80 \\
\hline$c_{2}$ & 1.00 & 1.00 \\
\hline$S_{2 x}$ & & 0.25 \\
\hline$S_{2 y}$ & & 1.10 \\
\hline$c_{3}$ & & 1.20 \\
\hline
\end{tabular}

\section{Generalization of the multi-arms technique}

In order to verify this idea by structure with more resonator numbers, a triple ring BSF is designed, simulated, optimized and fabricated on the similar substrate. The design and the fabricated filters are show in Figs. 14 and 15. The filter dimensions are presented in Table I. The filter consists of tree open-loop ring resonators, which are etched on the substrate and vertically overlapped and connected to each other through $50 \Omega$ microstrip feed. Fig. 16 shows the comparison between the simulated and measured results. Through optimizing the dimensions of the three ringresonators and choosing the suitable separation distances, enhanced rejection level, wide stopband and multiband features have been achieved. Additionally, the size of the proposed filter is very compact without using any shunt stub or defected ground structure cells. 


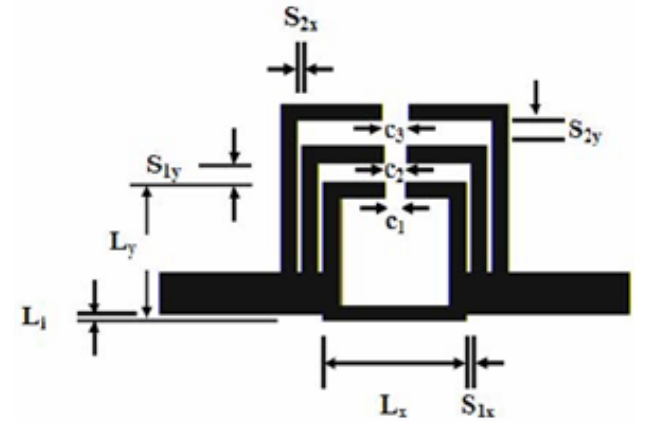

Fig.14. Physical layout of proposed triple rings BSF (Sample II).

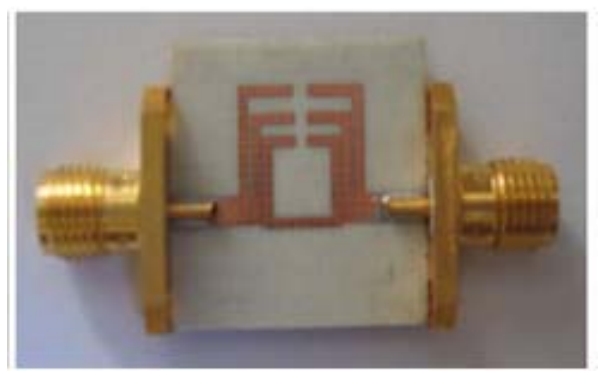

Figure15. Photograph of the fabricated BSF (Sample II).

The proposed filter has the potential of applying to $\mathrm{RF} /$ microwave integrated circuits. The total area of the filter is $\left(0.368 \lambda_{\mathrm{g}} \times 0.245 \lambda_{\mathrm{g}}\right)$, where $\lambda_{\mathrm{g}}=75 \mathrm{~mm}$. The calculated value of roll-off rate using the below equation amounts approximately $\xi_{\text {sup }}=85 \mathrm{~dB} / \mathrm{GHz}$.

$$
\xi_{\text {sup }}=\frac{\alpha_{\text {max }}-\alpha_{\text {min }}}{f_{0}-f_{c}}
$$

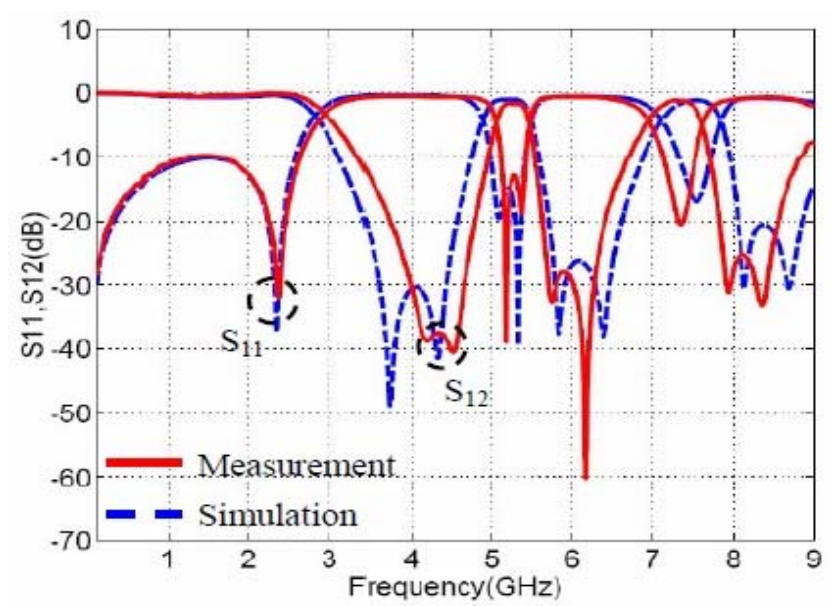

Figure 16. Measured and simulated scattering S-parameter results of triple rings-BSF (Sample II).

Table 2 gives a performance comparison between some reported BSFs and this work in terms of 3-dB cutoff frequency $\mathrm{f}_{\mathrm{c} \text {-inf }}$, resonance frequency $\mathrm{f}_{0}, \mathrm{BW}$, selectivity and circuit size. The presented BSF shows good performances such as wide stopband, sharpness and compact size.
Table 2. Comparison with some reported BSFs.

\begin{tabular}{|c|c|c|c|c|c|}
\hline Filter Topology & $\begin{array}{c}\mathrm{f}_{\mathrm{c}-\text { inf }} \\
(\mathrm{GHz})\end{array}$ & $\begin{array}{c}\mathrm{f}_{\mathrm{o}} \\
(\mathrm{GHz})\end{array}$ & $\begin{array}{c}\mathrm{BW} \\
(\mathrm{MHz})\end{array}$ & $\begin{array}{c}\xi_{\text {suup }} \\
(\mathrm{dB} / \mathrm{GHz})\end{array}$ & $\begin{array}{c}\text { Size } \\
\left(\mathrm{mm}^{2}\right)\end{array}$ \\
\hline$[1]$ & 0.720 & 0.725 & 20 & 570 & $33 \times 16$ \\
\hline$[2]$ & 3.4 & 3.5 & 500 & 45 & $50 \times 70$ \\
\hline$[5]$ & 2.1 & 2.5 & 900 & 85 & $140 \times 30$ \\
\hline$[7]$ & 5.1 & 5.5 & 500 & 29 & $25 \times 15$ \\
\hline$[26]$ & 2.38 & 2.426 & 300 & 566.7 & $20 \times 15$ \\
\hline Proposed filter & 2.8 & 4 & 1600 & 85 & $15 \times 15$ \\
\hline
\end{tabular}

\section{Conclusions}

In this paper, the design and implementation of a compact cascaded BSFs with a multi-band features, using double and triple identical C-open-loop-resonators has been presented. On the other hand an extracted new multi-band BSFs with separate stopband and sharp transition domains are designed and optimized and fabricated. The both novel multi-band filters consist of consist of several open-loop ring resonators placed vertically overlapping. Employing this technique, very compact filter topologies with design flexibility and excellent results are achieved. The size of the proposed multi-band filter using vertically overlapping method decreases $45 \%$ compared to the previous BSF using cascaded C-ring resonators. Furthermore, the filter achieves acceptable and separate multiple frequency bands. In order to show the validity of the approach, used method, the two filters were simulated, optimized and fabricated. The measurements show good consistency with the simulations. The slight discrepancy between both results can be attributed to unexpected tolerance errors in fabrication and manual welding inaccuracies. Such topologies can be easily integrated with RF systems which require a very compact and multi-band filter.

\section{Acknowledgements}

The author thanks the German Research Foundation (DFG) for financial support. The author thanks M.Sc. Eng. Sonja Boutejdar, Mehdi Boutejdar, Karim Boutjdir, Mohamed Boutejdar for their assistant and help and Mr. Harald Dempewolf, the lab manager of the Institute for Electronics, Signal Processing and Communication (IIKT) at the University of Magdeburg, Germany, for his support.

\section{References}

[1] S. Vegesna, M.A. Saed, Compact Two-Layer Microstrip Bandpass Filters Using Broadside-Coupled Resonators, Progress In Electromagnetics Research B 37: 81-102, 2012.

[2] J.M. Patin, N.R. Labadie, S.K. Sharma, Investigations on an H-Fractal Wideband Microstrip Filter with MultiPassbands and a Tuned Notch Band, Progress In Electromagnetics Research B 22: 285-303, 2010.

[3] L. Liu, R. Jin, X. Bai, Y. Li, X. Liang, J. Geng, C. He, A tri-band bandstop filter with sharp rejection and 
controllable bandstop frequencies, Antennas and Propagation \& USNC/URSI National Radio Science Meeting, 2015 IEEE International Symposium on, pp. $2543-2544,2015$

[4] A. Boutejdar, W. Abd Ellatif, A.A. Ibrahim, M. Challal, A simple transformation from lowpass to bandpass filter using a new quasi-arrow head defected ground structure resonator and gap-J-inverter, Microwave and Optical Technology Letters 58: 947-953, 2016.

[5] L.-C. Tsai, C.-W. Hsue, Dual-band bandpass filters using equal-length coupled-serial-shunted lines and ZTransform technique, IEEE Trans. Microwave Theory Tech. 52: 1111-1117, 2004.

[6] L.-C. Tsai, M.-L. Lee, Design of Low-pass Filters Using Three-and Two-section Stubs, Electron Devices and Solid-State Circuits, EDSSC 2007. IEEE Conference on , pp. 729-732, 2007.

[7] A. Boutejdar, A.A. Ibrahim, E.P. Burte, Design of 5 GHz-Compact Reconfigurable DGS-Bandpass Filter Using Varactor-diode Device and Coupling Matrix Technique, Microwave and Optical Technology Letters 58: 304-309, 2016.

[8] A. Boutejdar, A. Omar, M. Al Sharkawy, A. Darwish, A Simple Transformation of Improved WLAN Band Pass to Low Pass Filter Using Defected Ground Structure (DGS), Defected Microstrip Structure (DMS) and Multilayer-Technique, Journal of Microwaves, Optoelectronics and Electronics and Electromagnetic Applications (JMOE) 12: 111-130, 2013.

[9] A. Boutejdar et. al., An Improvement of Defected Ground Structure Lowpass/Bandpass Filters Using HSlot Resonators and Coupling Matrix Method, Journal of Microwaves, Optoelectronics and Electronics and Electromagnetic Applications (JMOE) 10: 295-307, 2011.

[10]L.-Y. Ren, Quad-band bandpass filter based on dualplane microstrip/DGS slot structure, Electron Lett. 46: 691-692, 2010.

[11]Z.M. Hejazi, Z. Ali, Multiband bandpass filters with suppressed harmonics using a novel defected ground structure, Microwave and Optical Technology Letters 56: 2726-273, 2014.

[12] A. Boutejdar, A. Elsherbini, A. Balalem, J. Machac, A. Omar, Design of New DGS Hairpin Microstrip Bandpass Filter Using Coupling Matrix Method, Progress In Electromagnetics Research Symposium, Prague, Czech Republic, pp.261-265, 2007.

[13] G. Karimi, F.K. Hamedani, H. Siahkamari, Ultra-wide stopband low-pass filter using symmetrical cascaded modified hairpin resonators, International Journal of RF and Microwave Computer-Aided Engineering 24: 314-321, 2014.

[14] A. Quendo, E. Ruis, A. Manchec, Y. Clavet, B. Potelon, J. -F. Favennec, C. Peson, Planar tri-band filter based on dualbehavior resonator (DBR), in Europ. Microw. Conf. Dig., Paris, 2005.
[15] M. Mokhtaari, J. Bornemann, S. Amari, Couplingmatrix design of dual/triple-band uni-planar filters, in IEEE MTT-S Int. Microw. Symp. Dig., San Francisco, CA, pp. 515-518, 2006.

[16] A. Boutejdar, Design of $5 \mathrm{GHz}$-compact reconfigurable DGS-bandpass filter using varactor-diode device and coupling matrix technique, Microwave Opt Technol Lett. 58: 304-309, 2016.

[17] A. Boutejdar et. al., Design of microstrip bandpass and lowpass filters using coupling matrix method and a new hairpin defected ground structure, Microwave Opt Technol Lett. 50: 2898-2901, 2008.

[18]P.S. Upare, On the Design of Compact Dual Band Rejection Filters using Circular Open Loop Resonators, International Journal of Engineering Science and Technology (IJEST) 4: 1886-1891, 2012.

[19] A. Boutejdar, N.M. Eltabit, A.A. Ibrahim, E.P. Burte, M.A. Abdalla, New Compact Dual Bandpass Filter Using Coupled Double-Ring Resonators and DGSTechnique, ACES Journal-The Applied Computational Electromagnetics 31: 132-137, 2016.

[20] A. Boutejdar, A. Elsherbini, S. Amari, M. Awida, A. Omar, Design of a novel microstrip bandstop filter using one compact C-open-loop resonator, in AsiaPacific Microwave Conf., Yokohama, Japan, 2006.

[21]A. Boutejdar, A New approach to Design Compact Tunable BPF starting from Simple LPF Topology Using a Single T-DGS-Resonator and Ceramic Capacitors, Microwave and Optical Technology Letters, Microwave Opt Technol Lett. 58: 1142-1148, 2016.

[22]A. Boutejdar, Theme der PhD thesis: Entwurf, Entwicklung und Optimierung von kompakten HFMikrostreifen-Filtern mittels Defected Ground Structure-Technik (DGS), doctorate degree (Dr. Eng.). At Otto-von-Guericke University, Magdeburg, November (2010).

[23] M. Awida, A. Boutejdar, A. Safwat, H. El-Hannawy A. Omar, Multi-Bandpass Filters Using Multi-Armed Split Ring Resontors with Direct Feed, IEEE MTT-S International Microwave Symposium, Honolulu, Hawaii, 2007.

[24] A. Boutejdar, N.M. Eltabit, A.A. Ibrahim, E.P. Burte, Design of Wide Stop Band L-Band LPF Based on DMS-DGS-Technique for Radar Applications, Hindawi Publishing Corporation International Journal of Microwave Science and Technology, Article ID 101602, 7-10, 2015.

[25] A. Boutejdar, Develop a BPF from a Compact DGS LPF, Microwave \& RF, Aug 30, 2016.

[26]A. Boutejdar, A. Omar, and E. Burte, Design and Optimization of New Compct Tunable 2.4-GHz Band Pass Filter Using Coupled $\lambda / 2$ Microstrip Open-Loop Resonators and MEMS-Switch Technique, Microwave and Optical Technology Letters 55: 2444-2450, 2013. 